\title{
Approximation of Modified Jakimovski-Leviatan-Beta Type Operators
}

\author{
MOHAmmad MursaleEN* AND MOHAmmad NASIRUZZAMAN
}

\begin{abstract}
In the present paper, we define Jakimovski-Leviatan type modified operators. We study some approximation results for these operators. We also determine the order of convergence in terms of modulus of continuity, Lipschitz functions, Peetre's K-functional, second order modulus of continuity and weighted modulus of continuity.
\end{abstract}

Keywords: Jakimovski-Leviatan operators, Korovkin's theorem, Modulus of continuity, Rate of convergence, $K$ functional, Weighted space

2010 Mathematics Subject Classification: 41A10, 41A25, 41A36.

\section{INTRODUCTION AND PRELIMINARIES}

Appell polynomials were introduced in 1880 (see [4]). In 1969, Jakimovski and Leviatan introduced an operators $P_{n}$ by using Appell polynomials [7]. The Appell polynomials are defined by the identity as follows:

$$
S(u) e^{u x}=\sum_{k=0}^{\infty} p_{k}(x) u^{k},
$$

for an analytic function in the disk $|u|<r(r>1)$ and $p_{n}(x)=\sum_{i=0}^{n} a_{i} \frac{x^{n-i}}{(n-i) !}(n \in \mathbb{N})$ taken $S(u)=\sum_{n=0}^{\infty} a_{n} u^{n}, S(1) \neq 0$. An exponential type the class of functions considerable on the semi-axis and satisfy the property $|f(x)| \leq \kappa e^{\gamma x}$, for some finite constants $\kappa, \gamma>0$ and denote the set of such functions by $E[0, \infty)$. The sequence of infinite sum of the operators $P_{n}$ is convergent and well-defined which are considered by the authors as follows [7]:

$$
P_{n}(f ; x)=\frac{e^{-n x}}{S(1)} \sum_{k=0}^{\infty} p_{k}(n x) f\left(\frac{k}{n}\right),
$$

for all $n \in \mathbb{N}$, where $n>\frac{\alpha}{\log r}$. In case of $\frac{a_{n}}{S(1)} \geq 0$ for all $n \in \mathbb{N}$, Wood [20] proved that the operator $P_{n}$ is positive on [0;1). For more results see also [13], [11] and [6]. They established that $\lim _{n \rightarrow \infty} P_{n}(f ; x) \rightarrow f(x)$, uniformly in each compact subset of $[0,1)$.

If $S(1)=1$ in (1.2) we get $p_{n}(x)=\frac{x^{n}}{n !}$, and we recover the well-known classical FavardSzász operators defined in 1950 by

$$
S_{n}(f ; x)=e^{-n x} \sum_{k=0}^{\infty} \frac{(n x)^{k}}{k !} f\left(\frac{k}{n}\right) .
$$

Received: August 13, 2018; In revised form: September 23, 2018; Accepted: October 1, 2018

*Corresponding author: M. Mursaleen; mursaleenm@gmail.com

DOI: $10.33205 / \mathrm{cma} .453284$ 
In the last quarter of twentieth century, the quantum calculus (also known as $q$ - calculus) was studied in $[8,12]$ (see $[3,14,15,18])$.

\section{CONSTRUCTION OF OpERATORS AND AuXiliary RESUlts}

In this paper, we define a Beta integral type modification of Jakimovski-Leviatan operators. We also introduce modified Jakimovski-Leviatan-Stancu type operators and obtain better approximation results. For $x \in[0, \infty), \quad p_{r}(x) \geq 0$ and $S(1) \neq 0$, we define

$$
J_{n}^{*}(f ; x)=\frac{e^{-n x}}{S(1)} \sum_{r=0}^{\infty} P_{r}(n x) \frac{1}{B(r+1, n)} \int_{0}^{\infty} \frac{t^{r}}{(1+t)^{r+n+1}} f(t) \mathrm{d} t,
$$

Lemma 2.1. If we take $e_{i}=t^{i-1}$ for $i=1,2,3$. Let $J_{n}^{*}(\cdot ; \cdot)$ be the operators given by (2.4). Then for all $x \in[0, \infty), \quad p_{r}(x) \geq 0$ and $S(1) \neq 0$, we have the following identities:

(1) $J_{n}^{*}\left(e_{1} ; x\right)=1$,

(2) $J_{n}^{*}\left(e_{2} ; x\right)=\left(\frac{n}{n-1}\right) x+\frac{1}{n-1}\left(\frac{S^{\prime}(1)}{S(1)}+1\right)$,

(3) $J_{n}^{*}\left(e_{3} ; x\right)=\frac{n^{2}}{(n-2)(n-1)} x^{2}+\frac{2 n}{(n-2)(n-1)}\left(\frac{S^{\prime}(1)}{S(1)}+2\right) x+\frac{1}{(n-2)(n-1)}\left(\frac{S^{\prime \prime}(1)}{S(1)}+\frac{S^{\prime}(1)}{S(1)}+2\right)$.

Proof. We can easily see that

$$
\sum_{r=0}^{\infty} P_{r}(n x)=S(1) e^{n x}
$$

$$
\sum_{r=0}^{\infty} r P_{r}(n x)=\left(S^{\prime}(1)+n S(1) x\right) e^{n x}
$$

$$
\sum_{r=0}^{\infty} r^{2} P_{r}(n x)=\left(S^{\prime \prime}(1)+2 n S^{\prime}(1) x+S^{\prime}(1)+n^{2} S(1) x^{2}\right) e^{n x}
$$

(1) By taking $f=e_{1}$

$$
\begin{aligned}
J_{n}^{*}\left(e_{1} ; x\right) & =\frac{e^{-n x}}{S(1)} \sum_{r=0}^{\infty} P_{r}(n x) \frac{1}{B(r+1, n)} \int_{0}^{\infty} \frac{t^{r}}{(1+t)^{r+n+1}} \mathrm{~d} t \\
& =\frac{e^{-n x}}{S(1)} \sum_{r=0}^{\infty} P_{r}(n x) \frac{B(r+1, n)}{B(r+1, n)} \\
& =1 .
\end{aligned}
$$

(2) By taking $f=e_{2}$ 


$$
\begin{aligned}
J_{n}^{*}\left(e_{2} ; x\right) & =\frac{e^{-n x}}{S(1)} \sum_{r=0}^{\infty} P_{r}(n x) \frac{1}{B(r+1, n)} \int_{0}^{\infty} \frac{t^{r+1}}{(1+t)^{r+n+1}} \mathrm{~d} t, \\
& =\frac{e^{-n x}}{S(1)} \sum_{r=0}^{\infty} P_{r}(n x) \frac{B(r+2, n-1)}{B(r+1, n)} \\
& =\frac{r+1}{n-1} \frac{e^{-n x}}{S(1)} \sum_{r=0}^{\infty} P_{r}(n x) \frac{B(r+1, n)}{B(r+1, n)} \\
& =\frac{1}{n-1}+\frac{1}{n-1} \frac{e^{-n x}}{S(1)} \sum_{r=0}^{\infty} r P_{r}(n x) \\
& =\frac{1}{n-1}+\frac{n}{n-1}\left(x+\frac{1}{n} \frac{S^{\prime}(1)}{S(1)}\right) .
\end{aligned}
$$

(3) By taking $f=e_{3}$

$$
\begin{aligned}
J_{n}^{*}\left(e_{2} ; x\right) & =\frac{e^{-n x}}{S(1)} \sum_{r=0}^{\infty} P_{r}(n x) \frac{1}{B(r+1, n)} \int_{0}^{\infty} \frac{t^{r+2}}{(1+t)^{r+n+1}} \mathrm{~d} t, \\
& =\frac{1}{(n-2)(n-1)} \frac{e^{-n x}}{S(1)} \sum_{r=0}^{\infty} P_{r}(n x)\left(r^{2}+3 r+2\right) \\
& =\frac{2}{(n-2)(n-1)}+\frac{3}{(n-2)(n-1)}\left(\frac{S^{\prime}(1)}{S(1)}+n x\right) \\
& +\frac{1}{(n-2)(n-1)}\left(\frac{S^{\prime \prime}(1)}{S(1)}+2 n x \frac{S^{\prime}(1)}{S(1)}+\frac{S^{\prime}(1)}{S(1)}+n x+n^{2} x^{2}\right) .
\end{aligned}
$$

Lemma 2.2. Take $\eta_{j}=\left(e_{i}-x\right)^{j}$ for $i=2, j=1,2$. Let $J_{n}^{*}(\cdot ; \cdot)$ be the operators given by (2.4). Then for all $x \in[0, \infty), \quad p_{r}(x) \geq 0$ and $S(1) \neq 0$, we have the following identities:

$$
\begin{aligned}
& 1^{\circ} \quad J_{n}^{*}\left(\eta_{1} ; x\right)=\frac{x}{n}+\frac{1}{n-1}\left(\frac{S^{\prime}(1)}{S(1)}+1\right) ; \\
& 2^{\circ} J_{n}^{*}\left(\eta_{2} ; x\right) \\
= & \frac{(n+2)}{(n-2)(n-1)} x^{2}+\frac{2 n}{(n-2)(n-1)}\left(\frac{2}{n}\left(\frac{S^{\prime}(1)}{S(1)}\right)+1\right) x+\frac{1}{(n-2)(n-1)}\left(\frac{S^{\prime \prime}(1)}{S(1)}+\frac{S^{\prime}(1)}{S(1)}+2\right) x .
\end{aligned}
$$
define

Let $\alpha, \beta \in \mathbb{R}$ such that $0 \leq \alpha<\beta$. Then for $x \in[0, \infty), \quad p_{r}(x) \geq 0$, and $S(1) \neq 0$, we

$$
J_{n}^{\alpha, \beta}(f ; x)=\frac{e^{-n x}}{S(1)} \sum_{r=0}^{\infty} P_{r}(n x) \frac{1}{B(r+1, n)} \int_{0}^{\infty} \frac{t^{r}}{(1+t)^{r+n+1}} f\left(\frac{n t+\alpha}{n+\beta}\right) \mathrm{d} t,
$$

Lemma 2.3. Take $e_{i}=t^{i-1}$ for $i=1,2,3$. Let $J_{n}^{\alpha, \beta}(\cdot ; \cdot)$ be the operators given by (2.8). Then for all $x \in[0, \infty), \quad p_{r}(x) \geq 0$ and $S(1) \neq 0$, we have the following identities:

$$
\begin{aligned}
& \text { (1) } J_{n}^{\alpha, \beta}\left(e_{1} ; x\right)=1 \\
& \text { (2) } J_{n}^{\alpha, \beta}\left(e_{2} ; x\right)=\frac{n^{4}}{(n+\beta)(n-1)} x+\frac{n}{(n+\beta)(n-1)}\left(\frac{S^{\prime}(1)}{S(1)}+1\right)+\frac{\alpha}{n+\beta} \\
& \text { (3) } J_{n}^{\alpha, \beta}\left(e_{3} ; x\right)=\frac{n^{2}}{(n+\beta)^{2}(n-2)(n-1)} x^{2}+\frac{2 n^{2}}{(n+\beta)^{2}(n-1)}\left\{\frac{n}{n-2}\left(\frac{S^{\prime}(1)}{S(1)}+2\right)+\alpha\right\} x \\
& +\frac{n^{2}}{(n+\beta)^{2}(n-2)(n-1)}\left(\frac{S^{\prime \prime}(1)}{S(1)}+\frac{S^{\prime}(1)}{S(1)}+2\right)+\frac{2 n \alpha}{(n+\beta)^{2}(n-1)}\left(\frac{S^{\prime}(1)}{S(1)}+1\right)+\frac{\alpha^{2}}{(n+\beta)^{2}} .
\end{aligned}
$$




\section{MAin Results}

We obtain the Korovkin type and weighted Korovkin type approximation theorems for the operators defined by (2.8).

Let $C_{B}[0, \infty)$ be the set of all bounded and continuous functions on $[0, \infty)$, which is a linear normed space with

$$
\|f\|_{C_{B}}=\sup _{x \geq 0}|f(x)|
$$

Let

and

$$
C_{\zeta}[0, \infty):=\left\{f \in C[0, \infty):|f(t)| \leq M(1+t)^{\zeta} \text { for some } M>0\right\},
$$

$$
H:=\left\{f \in C[0, \infty): \frac{f(x)}{1+x^{2}} \quad \text { is convergent as } x \rightarrow \infty\right\} .
$$

Theorem 3.1. Let $x \in[0, \infty), \quad f \in C_{\zeta}[0, \infty) \cap H$ with $\zeta \geq 2$. Then for $p_{r}(x) \geq 0, \quad S(1) \neq 0$, the operators $J_{n}^{\alpha, \beta}(\cdot ; \cdot)$ defined by $(2.8)$ satisfy

$$
\lim _{n \rightarrow \infty} J_{n}^{\alpha, \beta}(f ; x) \rightarrow f(x)
$$

uniformly on each compact subset of $[0, \infty)$.

Proof. The proof is based on Lemma 2.3 and well known Korovkin's theorem regarding the convergence of a sequence of linear positive operators. So it is enough to prove the conditions

$$
\lim _{n \rightarrow \infty} J_{n}^{\alpha, \beta}\left(\left(e_{i} ; x\right)=x^{i-1}, \quad i=1,2,3 \text { as } n \rightarrow \infty\right.
$$

uniformly on $[0, \infty]$.

Clearly $\frac{1}{n} \rightarrow 0, \quad(n \rightarrow \infty)$ we have

$$
\lim _{n \rightarrow \infty} J_{n}^{\alpha, \beta}\left(e_{2} ; x\right)=x, \quad \lim _{n \rightarrow \infty} J_{n}^{\alpha, \beta}\left(e_{3} ; x\right)=x^{2} .
$$

This completes the proof.

In the space $[0, \infty)$, following Gadžiev $[9,10,17]$, we recall the weighted spaces of the functions for which the analogous of the Korovkin theorem holds (see also $[1,5,19])$.

Let $x \rightarrow \phi(x)$ be a continuous and strictly increasing function and $\varrho(x)=1+\phi^{2}(x)$, $\lim _{x \rightarrow \infty} \varrho(x)=\infty$. Let $B_{\varrho}[0, \infty)$ be a set of functions defined on $[0, \infty)$ and satisfying

$$
|f(x)| \leq M_{f} \varrho(x),
$$

where $M_{f}$ is a constant depending only on $f$. Its subset of continuous functions will be denoted by $C_{\varrho}[0, \infty)$, i.e., $C_{\varrho}[0, \infty)=B_{\varrho}[0, \infty) \cap C[0, \infty)$. It is well known that a sequence of linear positive operators $\left\{J_{n}^{\alpha, \beta}\right\}_{n \geq 1}$ maps $C_{\varrho}[0, \infty)$ into $B_{\varrho}[0, \infty)$ if and only if

$$
\left|L_{n}(\varrho ; x)\right| \leq K \varrho(x),
$$

where $x \in[0, \infty)$ and $K$ is a positive constant. Note that $B_{\varrho}[0, \infty)$ is a normed space with the norm

$$
\|f\|_{\varrho}=\sup _{x \geq 0} \frac{|f(x)|}{\varrho(x)} .
$$

Finally, let $C_{\varrho}^{0}[0, \infty)$ be a subset of $C_{\varrho}[0, \infty)$ such that the limit

$$
\lim _{n \rightarrow \infty} \frac{f(x)}{\varrho(x)}=K_{f}
$$


exists and is finite.

Let $B[0,1]$ be the space of all bounded functions on $[0,1]$ and $C[0,1]$ be the space of all functions $f$ continuous on $[0,1]$ equipped with norm

$$
\|f\|_{\infty}=\sup _{x \in[0,1]}|f(x)|, \quad f \in C[0,1] .
$$

The famous Korovkin's theorems state as follows:

Theorem 3.2 (cf. [16]). Let $\left\{L_{n}\right\}_{n \geq 1}$ be the sequence of linear positive operators acting from $C[0,1]$ into $B[0,1]$. Then

$$
\lim _{n \rightarrow \infty}\left\|L_{n}\left(t^{k} ; x\right)-x^{k}\right\|_{\infty}=0(k=0,1,2),
$$

if and only if for all $f \in C[0,1]$

$$
\lim _{n \rightarrow \infty}\left\|L_{n}(f(t) ; x)-f\right\|_{\infty}=0 .
$$

Theorem 3.3. Let $\left\{J_{n}^{\alpha, \beta}\right\}_{n \geq 1}$ be the sequence of linear positive operators acting from $C_{\varrho}[0, \infty)$ into $B_{\varrho}[0, \infty)$ satisfies the conditions

$$
\lim _{n \rightarrow \infty}\left\|J_{n}^{\alpha, \beta}\left(\varphi^{i-1}(t) ; x\right)-\varphi^{i-1}(x)\right\|_{\varrho}=0(i=1,2,3)
$$

then for any function $f \in C_{\varrho}^{0}[0, \infty)$,

$$
\lim _{n \rightarrow \infty}\left\|J_{n}^{\alpha, \beta}(f(t) ; x)-f\right\|_{\varrho}=0 .
$$

Proof. For the completeness, we give some sketch of the proof for the version which will be used in our next result. Consider $\varphi(x)=x, \varrho(x)=1+x^{2}$, and

$$
\left\|J_{n}^{\alpha, \beta}\left(e_{i} ; x\right)-x^{i-1}\right\|_{\varrho}=\sup _{x \geq 0} \frac{\left|J_{n}^{\alpha, \beta}\left(e_{i} ; x\right)-x^{i-1}\right|}{1+x^{2}} .
$$

Then for $i=1,2,3$ it is easily proved that

$$
\lim _{n \rightarrow \infty}\left\|J_{n}^{\alpha, \beta}\left(e_{i} ; x\right)-x^{i-1}\right\|_{\varrho}=0 .
$$

Hence by using the above Theorem 3.2, for any function $f \in C_{\varrho}^{0}\left(\mathbb{R}^{+}\right)$, we get

$$
\lim _{n \rightarrow \infty}\left\|J_{n}^{\alpha, \beta}(f(t) ; x)-f\right\|_{\varrho}=0 .
$$

Theorem 3.4. Let $x \in[0, \infty), \quad f \in C_{\varrho}^{0}[0, \infty)$ with $\varrho(x)=1+x^{2}$. Then for $p_{r}(x) \geq 0, \quad S(1) \neq 0$, we have

$$
\lim _{n \rightarrow \infty}\left\|J_{n}^{\alpha, \beta}(f ; x)-f\right\|_{\varrho} \rightarrow 0 .
$$

Proof. Using Theorem 3.3 for $\varphi(x)=x$ and $\varrho(x)=1+x^{2}$, we consider

$$
\left\|J_{n}^{\alpha, \beta}\left(e_{i} ; x\right)-x^{i-1}\right\|_{\varrho}=\sup _{x \geq 0} \frac{\left|J_{n}^{\alpha, \beta}\left(e_{i} ; x\right)-x^{i-1}\right|}{1+x^{2}},
$$

for $i=1,2,3$.

According to Lemma 2.3 for $i=1$, it is obvious that $\left|J_{n}^{\alpha, \beta}\left(e_{1} ; x\right)-1\right| \rightarrow 0$, and therefore

$$
\lim _{n \rightarrow \infty}\left\|J_{n}^{\alpha, \beta}\left(e_{1} ; x\right)-1\right\|_{\varrho}=0 \text {. }
$$


For $i=2$

$$
\begin{aligned}
\sup _{x \geq 0} \frac{\left|J_{n}^{\alpha, \beta}\left(e_{2} ; x\right)-t\right|}{1+x^{2}} & \leq\left|\frac{n^{2}}{(n+\beta)(n-1)}-1\right| \sup _{x \geq 0} \frac{x}{1+x^{2}} \\
& +\left|\frac{n}{(n+\beta)(n-1)}\left(\frac{S^{\prime}(1)}{S(1)}+1\right)+\frac{\alpha}{n+\beta}\right| \sup _{x \geq 0} \frac{1}{1+x^{2}} .
\end{aligned}
$$

Therefore

$$
\lim _{n \rightarrow \infty}\left\|J_{n}^{\alpha, \beta}\left(e_{2} ; x\right)-x\right\|_{\varrho}=0
$$

For $i=3$

$$
\begin{aligned}
\sup _{x \geq 0} \frac{J_{n}^{\alpha, \beta}\left(e_{3} ; x\right)-x^{2} \mid}{1+x^{2}} & \leq\left|\frac{n^{4}}{(n+\beta)^{2}(n-2)(n-1)}-1\right| \sup _{x \geq 0} \frac{x^{2}}{1+x^{2}} \\
& +\left|\frac{2 n^{2}}{(n+\beta)^{2}(n-2)(n-1)}\left\{\frac{n}{n-2}\left(\frac{S^{\prime}(1)}{S(1)}+2\right)+\alpha\right\}\right| \sup _{x \geq 0} \frac{x}{1+x^{2}} \\
& +\mid \frac{n^{2}}{(n+\beta)^{2}(n-2)(n-1)}\left(\frac{S^{\prime \prime}(1)}{S(1)}+\frac{S^{\prime}(1)}{S(1)}+2\right) \\
& +\frac{2 n \alpha}{(n+\beta)^{2}(n-1)}\left(\frac{S^{\prime}(1)}{S(1)}\right)+\frac{\alpha^{2}}{(n+\beta)^{2}} \mid \sup _{x \geq 0} \frac{1}{1+x^{2}} .
\end{aligned}
$$

Hence we have

$$
\lim _{n \rightarrow \infty}\left\|J_{n}^{\alpha, \beta}\left(e_{3} ; x\right)-x^{2}\right\|_{\varrho}=0 .
$$

Which completes the proof of Korovkin's type theorem.

\section{Rate of Convergence}

Here we calculate the rate of convergence of operators (2.8) by means of modulus of continuity and Lipschitz type functions.

Let $f \in C_{B}[0, \infty]$ be the space of all bounded and uniformly continuous functions on $[0, \infty)$ and $x \geq 0$. Then for $\delta>0$, the modulus of continuity of $f$ denoted by $\omega(f, \delta)$ gives the maximum oscillation of $f$ in any interval of length not exceeding $\delta>0$ and it is given by

$$
\omega(f, \delta)=\sup _{|t-x| \leq \delta}|f(t)-f(x)|, \quad t \in[0, \infty) .
$$

It is known that $\lim _{\delta \rightarrow 0+} \omega(f, \delta)=0$ for $f \in C_{B}[0, \infty)$ and for any $\delta>0$ one has

$$
|f(t)-f(x)| \leq\left(\frac{|t-x|}{\delta}+1\right) \omega(f, \delta) .
$$

Take $\mu_{j}=\left(e_{i}-x\right)^{j}$ for $i=2, j=1,2$ and in the sequel we use the following notations:

$$
\delta_{n}^{\alpha, \beta}=\sqrt{J_{n}^{\alpha, \beta}\left(\mu_{2} ; x\right)},
$$


Here

$$
J_{n}^{\alpha, \beta}\left(\mu_{j} ; x\right)=\left\{\begin{array}{l}
\left(\frac{n^{2}}{(n+\beta)(n-1)}-1\right) x+\frac{n}{(n+\beta)(n-1)}\left(\frac{S^{\prime}(1)}{S(1)}+1\right)+\frac{\alpha}{n+\beta} \\
\quad \text { for } j=1,0<\alpha<\beta, \alpha, \beta \in \mathbb{R} \\
\left(\frac{n^{4}}{(n+\beta)^{2}(n-2)(n-1)}-\frac{2 n^{2}}{(n+\beta)(n-1)}+1\right) x^{2} \\
+\left[\frac{2 n^{2}}{(n+\beta)^{2}(n-1)}\left\{\frac{n}{n-2}\left(\frac{S^{\prime}(1)}{S(1)}+2\right)+\alpha\right\}\right. \\
\left.-\frac{2 n}{(n+\beta)(n-1)}\left(\frac{S^{\prime}(1)}{S(1)}+1\right)+\frac{2 \alpha}{n+\beta}\right] x \\
+\frac{n^{2}}{(n+\beta)^{2}(n-2)(n-1)}\left(\frac{S^{\prime \prime}(1)}{S(1)}+\frac{S^{\prime}(1)}{S(1)}+2\right) \\
+\frac{2 n \alpha}{(n+\beta)^{2}(n-1)}\left(\frac{S^{\prime}(1)}{S(1)}+1\right)+\frac{\alpha^{2}}{(n+\beta)^{2}} \\
\text { for } j=2,0<\alpha<\beta, \alpha, \beta \in \mathbb{R}
\end{array}\right.
$$$$
\text { when } \alpha=\beta=0 \text {, then } \delta_{n}^{\alpha, \beta} \text { is reduced to } \delta_{n}^{*}=\sqrt{J_{n}^{*}\left(\eta_{2} ; x\right)} \text {. }
$$

Theorem 4.5. For $x \in[0, \infty), \quad f \in C_{B}[0, \infty)$ the operators $J_{n}^{\alpha, \beta}(\cdot ; \cdot)$ defined by (2.8) satisfying:

$$
\left|J_{n}^{\alpha, \beta}(f ; x)-f(x)\right| \leq 2 \omega\left(f ; \delta_{n}^{\alpha, \beta}\right),
$$

where $n \in \mathbb{N}, \quad p_{r}(x) \geq 0, \quad S(1) \neq 0$ and $\delta_{n}^{\alpha, \beta}$ is defined in (4.11).

Proof. For our sequence of positive linear operators $\left\{J_{n}^{\alpha, \beta}(. ;).\right\}$ we have

$$
\begin{aligned}
J_{n}^{\alpha, \beta}(f ; x)-f(x) & =J_{n}^{\alpha, \beta}(f ; x)-f(x) J_{n}^{\alpha, \beta}(1 ; x) \\
& =J_{n}^{\alpha, \beta}(f(t)-f(x) ; x) \\
& \leq J_{n}^{\alpha, \beta}(|f(t)-f(x)| ; x),
\end{aligned}
$$

since $J_{n}^{\alpha, \beta}(1 ; x)=1$. From (4.9) and (4.10) easily we get

$$
\begin{aligned}
\left|J_{n}^{\alpha, \beta}(f ; x)-f(x)\right| & \leq J_{n}^{\alpha, \beta}\left(1+\frac{|t-x|}{\delta} ; x\right) \omega(f ; \delta) \\
& =\left(1+\frac{1}{\delta} J_{n}^{\alpha, \beta}(|t-x| ; x)\right) \omega(f ; \delta) .
\end{aligned}
$$

Cauchy-Schwarz inequality give us

$$
J_{n}^{\alpha, \beta}(|t-x| ; x) \leq J_{n}^{\alpha, \beta}(1 ; x)^{\frac{1}{2}} J_{n}^{\alpha, \beta}\left((t-x)^{2} ; x\right)^{\frac{1}{2}}
$$

so that

$$
\left|J_{n}^{\alpha, \beta}(f ; x)-f(x)\right| \leq\left(1+\frac{1}{\delta} J_{n}^{\alpha, \beta}\left((t-x)^{2} ; x\right)^{\frac{1}{2}}\right) \omega(f ; \delta) .
$$

Finally, putting $\delta=\delta_{n}^{\alpha, \beta}=\sqrt{J_{n}^{\alpha, \beta}\left(\mu_{2} ; x\right)}$ we get the assertion. 
Remark 4.1. Choosing $\delta=\frac{1}{n+\beta}$ in (4.13) we obtain the following estimate

$$
\left|J_{n}^{\alpha, \beta}(f ; x)-f(x)\right| \leq\left(1+(n+\beta) \delta_{n}^{\alpha, \beta}\right) \omega\left(f ; \frac{1}{n+\beta}\right),
$$

where $\delta_{n}^{*}$ defined in (4.11).

Remark 4.2. For $\alpha=\beta=0$ the corresponding estimate for the sequence of positive linear operators $\left\{J_{n}^{\alpha, \beta}\right\}$ is reduced to $\left\{J_{n}^{*}\right\}$ defined by (2.4) which can take the form as

$$
\left|J_{n}^{*}(f ; x)-f(x)\right| \leq 2 \omega\left(f ; \delta_{n}^{*}\right),
$$

where $\delta_{n}^{*}=\sqrt{\left.J_{n}^{*}\left(\eta_{2} ; x\right)\right)}$.

Now we give the rate of convergence of the operators $J_{n}^{\alpha, \beta}(f ; x)$ defined in (2.8) in terms of the elements of the usual Lipschitz class $\operatorname{Lip}_{M}(\nu)$. Let $f \in C_{B}[0, \infty), M>0$ and $0<\nu \leq 1$. The class $\operatorname{Lip}_{M}(\nu)$ is defined as

$$
\operatorname{Lip}_{M}(\nu)=\left\{f:\left|f\left(\zeta_{1}\right)-f\left(\zeta_{2}\right)\right| \leq M\left|\zeta_{1}-\zeta_{2}\right|^{\nu}\left(\zeta_{1}, \zeta_{2} \in[0, \infty)\right)\right\} .
$$

Theorem 4.6. Suppose $x \in[0, \infty), \quad f \in \operatorname{Lip}_{M}(\nu)$ with $(M>0, \quad 0<\nu \leq 1)$. Then operators $J_{n}^{\alpha, \beta}(\cdot ; \cdot)$ defined by (2.8) satisfying:

$$
\left|J_{n}^{\alpha, \beta}(f ; x)-f(x)\right| \leq M\left(\delta_{n}^{\alpha, \beta}\right)^{\nu / 2},
$$

where $\delta_{n}^{\alpha, \beta}$ is defined in (4.11).

Proof. Use (4.16) and apply Hölder's inequality

$$
\begin{aligned}
\left|J_{n}^{\alpha, \beta}(f ; x)-f(x)\right| & \leq\left|J_{n}^{\alpha, \beta}(f(t)-f(x) ; x)\right| \\
& \leq J_{n}^{\alpha, \beta}(|f(t)-f(x)| ; x) \\
& \leq M J_{n}^{\alpha, \beta}\left(|t-x|^{\nu} ; x\right) .
\end{aligned}
$$

Therefore

$$
\begin{aligned}
\mid J_{n}^{\alpha, \beta}(f ; x) & -f(x) \mid \\
& \leq M \frac{e^{-n x}}{S(1)} \sum_{r=0}^{\infty} P_{r}(n x) \frac{1}{B(r+1, n)} \int_{0}^{\infty} \frac{t^{r}}{(1+t)^{r+n+1}}|t-x|^{\nu} \mathrm{d} t \\
& =M \frac{e^{-n x}}{S(1)}\left(\sum_{r=0}^{\infty} P_{r}(n x) \frac{1}{B(r+1, n)}\right)^{\frac{2-\nu}{2}} \\
& \times\left(P_{r}(n x) \frac{1}{B(r+1, n)}\right)^{\frac{\nu}{2}} \int_{0}^{\infty} \frac{t^{r}}{(1+t)^{r+n+1}|t-x|^{\nu} \mathrm{d} t} \\
& \leq M\left(\frac{e^{-n x}}{S(1)} \sum_{r=0}^{\infty} P_{r}(n x) \frac{1}{B(r+1, n)} \int_{0}^{\infty} \frac{t^{r}}{(1+t)^{r+n+1}} \mathrm{~d} t\right)^{\frac{2-\nu}{2}} \\
& \times\left(\frac{e^{-n x}}{S(1)} \sum_{r=0}^{\infty} P_{r}(n x) \frac{1}{B(r+1, n)} \int_{0}^{\infty} \frac{t^{r}}{(1+t)^{r+n+1}}|t-x|^{2} \mathrm{~d} t\right)^{\frac{\nu}{2}} \\
& =M J_{n}^{\alpha, \beta}\left(\mu_{2} ; x\right)^{\frac{\nu}{2}} .
\end{aligned}
$$

This completes the proof. 
Let

$$
C_{B}^{2}[0, \infty)=\left\{g \in C_{B}[0, \infty): g^{\prime}, g^{\prime \prime} \in C_{B}[0, \infty)\right\}
$$

with the norm

$$
\|g\|_{C_{B}^{2}[0, \infty)}=\|g\|_{C_{B}[0, \infty)}+\left\|g^{\prime}\right\|_{C_{B}[0, \infty)}+\left\|g^{\prime \prime}\right\|_{C_{B}[0, \infty)}
$$

where

$$
\|g\|_{C_{B}[0, \infty)}=\sup _{x \in[0, \infty)}|g(x)| .
$$

Theorem 4.7. Let $x \in[0, \infty)$ and $J_{n}^{\alpha, \beta}(\cdot ; \cdot)$ be the operator defined by (2.8). Then for any $g \in$ $C_{B}^{2}[0, \infty)$, we have

$$
\left|J_{n}^{\alpha, \beta}(f ; x)-f(x)\right| \leq \frac{1}{2} \delta_{n}^{\alpha, \beta}\left(2+\delta_{n}^{\alpha, \beta}\right)\|g\|_{C_{B}^{2}[0, \infty)},
$$

where $n \in \mathbb{N}, \quad p_{r}(x) \geq 0, S(1) \neq 0$ and $\delta_{n}^{\alpha, \beta}$ is defined in (4.11).

Proof. Let $g \in C_{B}^{2}[0, \infty)$. Then by using the generalized mean value theorem in the Taylor series expansion we have

$$
g(t)=g(x)+g^{\prime}(x)(t-x)+g^{\prime \prime}(\psi) \frac{(t-x)^{2}}{2},
$$

which follows

$$
|g(t)-g(x)| \leq M_{1}|t-x|+\frac{1}{2} M_{2}(t-x)^{2},
$$

where by using the result of (4.18) and (4.19) we have

$$
\begin{gathered}
M_{1}=\sup _{x \in[0, \infty)}\left|g^{\prime}(x)\right|=\left\|g^{\prime}\right\|_{C_{B}[0, \infty)} \leq\|g\|_{C_{B}^{2}[0, \infty)}, \\
M_{2}=\sup _{x \in[0, \infty)}\left|g^{\prime \prime}(x)\right|=\left\|g^{\prime \prime}\right\|_{C_{B}[0, \infty)} \leq\|g\|_{C_{B}^{2}[0, \infty)},
\end{gathered}
$$

again from 4.18 , we have

$$
|g(t)-g(x)| \leq\left(|t-x|+\frac{1}{2}(t-x)^{2}\right)\|g\|_{C_{B}^{2}[0, \infty)} .
$$

Since

$$
\left|J_{n}^{\alpha, \beta}(g, x)-g(x)\right|=\left|J_{n}^{\alpha, \beta}(g(t)-g(x) ; x)\right| \leq J_{n}^{\alpha, \beta}(|g(t)-g(x)| ; x),
$$

and also

$$
J_{n}^{\alpha, \beta}(|t-x| ; x) \leq J_{n}^{\alpha, \beta}\left((t-x)^{2} ; x\right)^{\frac{1}{2}}=\delta_{n}^{\alpha, \beta}
$$

we get

$$
\begin{aligned}
\left|J_{n}^{\alpha, \beta}(g ; x)-g(x)\right| & \leq\left(J_{n}^{\alpha, \beta}(|t-x| ; x)+\frac{1}{2} J_{n}^{\alpha, \beta}\left((t-x)^{2} ; x\right)\right)\|g\|_{C_{B}^{2}[0, \infty)} \\
& \leq \frac{1}{2} \delta_{n}^{\alpha, \beta}\left(2+\delta_{n}^{\alpha, \beta}\right)\|g\|_{C_{B}^{2}[0, \infty)} .
\end{aligned}
$$

This completes the proof. 
The Peetre's $K$-functional is defined by

$$
K_{2}(f, \delta)=\inf _{C_{B}^{2}[0, \infty)}\left\{\left(\|f-g\|_{C_{B}[0, \infty)}+\delta\left\|g^{\prime \prime}\right\|_{C_{B}^{2}[0, \infty)}\right): g \in \mathcal{W}^{2}\right\}
$$

where

$$
\mathcal{W}^{2}=\left\{g \in C_{B}[0, \infty): g^{\prime}, g^{\prime \prime} \in C_{B}[0, \infty)\right\} .
$$

There exits a positive constant $C>0$ such that $K_{2}(f, \delta) \leq C \omega_{2}\left(f, \delta^{1 / 2}\right), \quad \delta>0$, where the second order modulus of continuity is given by

$$
\omega_{2}\left(f, \delta^{1 / 2}\right)=\sup _{0<h<\delta^{1 / 2}} \sup _{x \in \mathbb{R}^{+}}|f(x+2 h)-2 f(x+h)+f(x)| .
$$

Theorem 4.8. Suppose $x \in[0, \infty), n \in \mathbb{N}$ and $f \in C_{B}[0, \infty)$. Then the operators $J_{n}^{\alpha, \beta}(\cdot ; \cdot)$ defined by (2.8) satisfying

$$
\left|J_{n}^{\alpha, \beta}(f ; x)-f(x)\right| \leq 2 M\left\{\omega_{2}\left(f ; \sqrt{\Delta_{n}^{\alpha, \beta}}\right)+\min \left(1, \Delta_{n}^{\alpha, \beta}\right)\|f\|_{C_{B}[0, \infty)}\right\},
$$

where $M$ is a positive constant, $p_{r}(x) \geq 0, \quad S(1) \neq 0$ and $\Delta_{n}^{\alpha, \beta}=\frac{\left(2+\delta_{n}^{\alpha, \beta}\right) \delta_{n}^{\alpha, \beta}}{4}$.

Proof. As previous we easily conclude that

$$
\begin{aligned}
\left|J_{n}^{\alpha, \beta}(f ; x)-f(x)\right| & \leq\left|J_{n}^{\alpha, \beta}(f-g ; x)\right|+\left|J_{n}^{\alpha, \beta}(g ; x)-g(x)\right|+|f(x)-g(x)|, \\
& \leq 2\|f-g\|_{C_{B}[0, \infty)}+\frac{\delta_{n}^{\alpha, \beta}}{2}\left(2+\delta_{n}^{\alpha, \beta}\right)\|g\|_{C_{B}^{2}[0, \infty)}, \\
& \leq 2\left(\|f-g\|_{C_{B}[0, \infty)}+\frac{\delta_{n}^{\alpha, \beta}}{4}\left(2+\delta_{n}^{\alpha, \beta}\right)\|g\|_{C_{B}^{2}[0, \infty)}\right) .
\end{aligned}
$$

By taking infimum over all $g \in C_{B}^{2}[0, \infty)$ and by using (4.20), we get

$$
\left|J_{n}^{\alpha, \beta}(f ; x)-f(x)\right| \leq 2 K_{2}\left(f ; \frac{\delta_{n}^{\alpha, \beta}\left(2+\delta_{n}^{\alpha, \beta}\right)}{4}\right) .
$$

Now for an absolute constant $M>0$ in [2] we use the relation

$$
K_{2}(f ; \delta) \leq M\left\{\omega_{2}(f ; \sqrt{\delta})+\min (1, \delta)\|f\|\right\} .
$$

This completes the proof.

\section{REFERENCES}

[1] T. Acar, Asymptotic formulas for generalized Szász-Mirakyan operators, Appl. Math. Comput., 263 (2015) 223239.

[2] A. Ciupa, A class of integral Favard-Szász type operators, Stud. Univ. Babeş-Bolyai, Math., 40 (1995) 39-47.

[3] W.A. Al-Salam, $q$-Appell polynomials. Ann. Mat. Pura Appl., 4 (1967) 31-45.

[4] P. Appell, Une classe de polynômes, Ann. Sci. École Norm. Sup., 9 (1880) 119-144.

[5] A. Aral, T. Acar, Weighted approximation by new Bernstein-Chlodowsky- Gadjiev operators, Filomat, 27 (2013) 371-380.

[6] C. Atakut, I. Büyükyazici, Approximation by modified integral type Jakimovski-Leviatan operators, Filomat, 30 (2016) 29-39.

[7] İ. Büyükyazıcı, H. Tanberkan, S. Serenbay, C. Atakut, Approximation by Chlodowsky type Jakimovski-Leviatan operators, Jour. Comput. Appl. Math., 259 (2014) 153-163.

[8] J. Choi, H.M. Srivastava, $q$-Extensions of a multivariable and multiparameter generalization of the Gottlieb polynomials in several variables, Tokyo J. Math., 37 (2014) 111-125.

[9] A. D. Gadzhiev, A problem on the convergence of a sequence of positive linear operators on unbounded sets, and theorems that are analogous to P. P. Korovkin's theorem. Dokl. Akad. Nauk SSSR (Russian), 218 (1974) 1001-1004. 
[10] A. D. Gadzhiev, Weighted approximation of continuous functions by positive linear operators on the whole real axis, Izv. Akad. Nauk Azerbaijan. SSR Ser. Fiz.-Tehn. Mat. Nauk (Russian), 5 (1975) 41-45.

[11] P. Gupta, P. N. Agarwal, Jakimovski-Leviatan operators of Durrmeyer type involving involving Appell polynomials, Turk J. Math., 42 (2018) 1457-1470.

[12] F.H. Jackson, On q-definite integrals, Quart. J. Pure Appl. Math., 41(15) (1910) 193-203.

[13] A. Jakimovski, D. Leviatan, Generalized Szasz operators for the approximation in the infinite interval. Mathematica (Cluj), 11 (34) (1969) 97-103.

[14] V. Kac., A. De Sole, On integral representations of $q$-gamma and $q$-beta functions, Rend. Mat. Acc. Lincei, 9 (200) $11-29$.

[15] M.E. Keleshteri, N.I. Mahmudov, A study on $q$-Appell polynomials from determinantal point of view, Appl. Math. Comp., 260 (2015) 351-369.

[16] P.P. Korovkin, Linear Operators And Approximation Theory, Hindustan Publ. Co. Delhi, 1960.

[17] G. V. Milovanović, M. Mursaleen., M. Nasiruzzaman, Modified Stancu type Dunkl generalization of SzászKantorovich operators, Rev. R. Acad. Cienc. Exactas Fis. Nat. Ser. A Math. RACSAM, 112(1) (2018) $135-151$.

[18] M. Mursaleen, K.J. Ansari, M. Nasiruzzaman, Approximation by $q$-analogue of Jakimovski-Leviatan operators involving $q$-Appell polynomials, Iran. J. Sci. Technol. Trans. Sci. 41 (2017) 891-900.

[19] A. Wafi, N. Rao, D. Rai, Appproximation properties by generalized-Baskakov-Kantrovich-Stancu type operators, Appl. Math. Inform. Sci. Lett., 4 (2016) 111-118.

[20] B. Wood, Generalized Szász operators for the approximation in the complex domain, SIAM J. Appl. Math., 17 (1969) 790-801.

DePARTMENT OF MATHEMATICS

Aligarh Muslim UNIVERSITY

ALIGARH-202002, INDIA

E-mail address: mursaleenmegmail . com

DEPARTMENT OF COMPUTER SCIENCE (SEST)

JAMIA HAMDARD UNIVERSITY

NEW DELHI-110062,

INDIA DEPARTMENT OF CIVIL ENGINEERING

JAmia Millia UniVersity

NEW DELHI-110025, INDIA

E-mail address: nasir3489@gmail.com 